\title{
How to choose a fuzzy similarity measure in decision-making?
}

Bernadette Bouchon-Meunier

LIP6, Sorbonne Université-CNRS, Paris, France, and

Giulianella Coletti

Universita degli Studi di Perugia, Perugia, Italy

\begin{abstract}
Purpose - The paper is dedicated to the analysis of fuzzy similarity measures in uncertainty analysis in general, and in economic decision-making in particular. The purpose of this paper is to explain how a similarity measure can be chosen to quantify a qualitative description of similarities provided by experts of a given domain, in the case where the objects to compare are described through imprecise or linguistic attribute values represented by fuzzy sets. The case of qualitative dissimilarities is also addressed and the particular case of their representation by distances is presented.

Design/methodology/approach - The approach is based on measurement theory, following Tversky's well-known paradigm.

Findings - A list of axioms which may or may not be satisfied by a qualitative comparative similarity between fuzzy objects is proposed, as extensions of axioms satisfied by similarities between crisp objects. They enable to express necessary and sufficient conditions for a numerical similarity measure to represent a comparative similarity between fuzzy objects. The representation of comparative dissimilarities is also addressed by means of specific functions depending on the distance between attribute values.

Originality/value - Examples of functions satisfying certain axioms to represent comparative similarities are given. They are based on the choice of operators to compute intersection, union and difference of fuzzy sets. A simple application of this methodology to economy is given, to show how a measure of similarity can be chosen to represent intuitive similarities expressed by an economist by means of a quantitative measure easily calculable. More detailed and formal results are given in Coletti and Bouchon-Meunier (2020) for similarities and Coletti et al. (2020) for dissimilarities.
\end{abstract}

Keywords Fuzzy sets, Similarity measures, Dissimilarity measure, Measurement theory, Qualitative choice

Paper type Research paper

\section{Introduction}

The purpose of this paper is to revisit results introduced in Coletti and Bouchon-Meunier (2019a) and developed in Coletti and Bouchon-Meunier (2020) in a more intuitive approach, enabling the reader to grasp the meaning of the formal results based on measurement theory with the aim of making a conscious choice between several possible measures. Such a choice is necessary because of the number of similarity measures and their fuzzy extensions existing in the literature, whose

(C) Bernadette Bouchon-Meunier and Giulianella Coletti. Published in Asian Journal of Economics and Banking. Published by Emerald Publishing Limited. This article is published under the Creative Commons Attribution (CC BY 4.0) licence. Anyone may reproduce, distribute, translate and create derivative works of this article (for both commercial and non-commercial purposes), subject to full attribution to the original publication and authors. The full terms of this licence may be seen at http:// creativecommons.org/licences/by/4.0/legalcode

JEL classification - $\mathrm{C} 00, \mathrm{C} 18, \mathrm{C} 10$,

MSC2020 classification - 91C05, 03E72, 03B52, 91C20

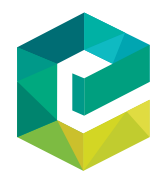

Asian Journal of Economics and Banking

Vol. 4 No. 3, 2020

pp. 37-48 2615-982 DOI 10.1108/AJEB-08-2020-0055 
properties cannot be directly apprehended by the user. We follow Tversky's paradigm based on measurement theory to define necessary and sufficient conditions for the existence of a particular class of fuzzy similarity measures, which we express in an intuitive formulation. Similarity measures, regarded as the representation of binary relations between objects expressing the idea that " $X$ is no more similar to $Y$ than $X^{\prime}$ is to $Y^{\prime}$ " have been extensively studied in statistics, data analysis, decision support systems, pattern recognition, clustering methods, information retrieval, case-based reasoning, similarity-based reasoning and may other fields. With the aim of helping the user to choose one of the possible measures, we have presented a qualitative assessment of similarity measures in Bouchon-Meunier et al. $(2009,2010)$ and we have defined a more general approach of properties of similarity measures based on measurement theory in Coletti and Bouchon-Meunier (2019b, 2020). In this paper, we focus on fuzzy similarity measures, used to evaluate the similarity between two objects which are ill-defined, imprecisely or linguistically described. The concept of similarity relation has first been introduced in Zadeh (1971) to represent a gradual relation between precise elements, which can be expressed linguistically by " $x$ is rather similar to $y$ " or " $x$ is very similar to $y$ ". The similarity between objects whose attribute values are characterised by fuzzy sets has then been explored, for instance by Costas and Nikos (1993), LeeKwang et al. (1994), Chen et al. (1995), Wang et al. (1995) and Fan and Xie (1999). We have introduced in Bouchon-Meunier et al. (1996), a more general classification of measures of comparison according to their elementary properties. In Coletti and Bouchon-Meunier (2019a) and more extensively in Coletti and Bouchon-Meunier (2020), we have proposed to discover empirically the qualitative conditions which we implicitly accept when we choose a given fuzzy similarity measure and we have highlighted the properties underlying well-known classes of fuzzy similarity measures.

In Coletti et al. (2020), we have investigated the case of qualitative dissimilarities and their representation by means of quantitative dissimilarity measures, focusing on distances that are the most popular dissimilarity measures.

In the sequence, we first present the background of the study, based on Tversky's model of similarities, illustrated by a paradigmatic example on Basel Accords, followed by the introduction of a comparative similarity and the main axioms that we consider. Then we present general classes of similarities that satisfy several of these axioms. We highlight independence axioms that are pointed out by Tversky (1977). We then develop these concepts on the illustrative example. We continue on the representation of comparative dissimilarities and we conclude the paper.

\section{Fuzzy similarity measures}

\subsection{Purpose of the study}

We focus our study on the similarity between objects described by imprecise or linguistic values of attributes, represented by fuzzy membership degrees. We consider comparative similarities, regarded as binary relations on pairs of objects, meaning that " $X$ is no more similar to $Y$ than $X^{\prime}$ is to $Y^{\prime \prime}$. We look for a representation of such comparative similarities by means of real-valued functions defining fuzzy similarity measures, with the purpose of a numerical management of similarity that preserves the essence of qualitative comparative similarities while allowing for the automation of evaluations. To achieve this goal, we identify the requirements underlying the choice of a fuzzy similarity measure on the basis of measurement theory, in the spirit of the formalisation of classic similarities given in Tversky (1977).

\subsection{Paradigmatic example}

To clarify our path, we will consider as an example the similarity between companies applying for credit from some European financial institution in accordance with the Basel Accords, under which the bank must set aside a part of its assets according to the risk of each loan granted. 
The banking and financial system considers the rating as the essential element to assess the financial reliability of a company. The rating is in fact a summary assessment of credit risk, it is a sort of "grade" that the banking system attributes to a company, based on the behaviour that the company maintains towards the system itself. In summary, it enables to classify credit applicants into classes of risk.

For the banking system, after Basel II (Basel Committee on Banking Supervision, 2001) and Basel III (Basel Committee on Banking Supervision, 2017), what matters is not so much the amount of a company's net debt, but rather the company's ability to pay the debts it has contracted with banks, suppliers and authorities. In other words, more value is given to the company's ability to meet its obligations on a regular basis than the level of debt exposure that weighs on the company.

To determine the rating, other parameters also concur, such as the capital solidity, the analyses related to the sector in which the company operates, the plan of medium- and longterm development presented by the company, the state of company liquidity and also the characteristics of the company management.

The bank summarises all the information in its possession and expresses an opinion on the reliability of the company through the rating.

To date, there is no single evaluation system; in fact, the rating may vary depending on the credit institution. However, the type of information analysed is similar for all banks and is:

- quantitative information;

- qualitative information; and

- performance information.

Now, on the basis of the foregoing and additional information obtained from Basel II and Basel III, for the purpose of calculating the bank rating, a company can be described by the following characteristics or features:

- $h_{1}$ : high quality (of the enterprise);

- $h_{2}$ : growing sector;

- $h_{3}$ : high budget;

- $h_{4}$ : low cost of interest paid;

- $h_{5}$ : compliance with the terms of repayment of the credits received;

- $h_{6}$ : failure to exceed the credit threshold available;

- $h_{7}$ : the breakdown of debts between short, medium and long term;

- $h_{8}$ : high value of the level of stocks and commercial credits of the company;

- $h_{9}$ : absence of current accounts inactive or with a negative balance; and

- $h_{10}$ : good use of credit lines.

By their nature, the characteristics $h_{i}$ are not of the Boolean type and can therefore belong to a company under study to a certain degree $x_{i}=\mu\left(h_{i}\right) \in[0,1]$.

It is natural to expect elements in one rating class to be more similar to each other than elements in different classes. This can also be used as a validity test of the rating calculation procedure done by the bank or an independent agency, However, as we will see, this depends on the choice of various factors, first of all, on the choice of the measure of similarity, or even of the class of similarity measures. Therefore this class must be chosen consciously, in the sense that it must be clear which rules are accepted when the similarity of two companies is measured through an element of the class. 
AJEB

4,3

\subsection{Framework}

The objects we consider are described by means of a set of attributes $\mathcal{H}=\left\{h_{1}, \ldots, h_{p}\right\}$ in such a way that the data set $\mathcal{Y}=[0,1]^{p}$ contains all possible descriptions. For every $X=\left\{x_{1}, \ldots, x_{p}\right\}$ $\in Y$, each $x_{i}$ is defined by the degree of membership $\mu_{X}\left(h_{i}\right) \in[0,1]$ of $h_{i}$ in object $X$. We consider the measure of $X$ defined as $m(X)=\sum_{u} \mu_{X}(u)$. We use the classic Gödel operators on fuzzy sets, i.e. the minimum for the intersection and the maximum for the union, and we define the complement $X^{c}$ of $X$ by the classic operation 1-x. Differences between fuzzy sets are defined as $X \backslash Y=X \cap Y^{c}$ with $k$-th component $\min \left\{x_{k}, 1-y_{k}\right\}$ and $Y X=X^{c} \cap Y$ with $k$-th component $\min \{1$ $\left.-x_{k}, y_{k}\right\}$, and the symmetric difference between $X$ and $Y$ as $X \Delta Y=(X \backslash Y) \cup(Y X)$.

\subsection{Comparative similarity}

We define a comparative similarity on $\mathcal{Y}^{2}$ as a binary relation denoted by $\preccurlyeq$, such that, for two pairs of objects $(X, Y)$ and $\left(X^{\prime}, Y^{\prime}\right) \in \mathcal{Y}^{2},(X, Y) \preccurlyeq\left(X^{\prime}, Y^{\prime}\right)$ means that $X$ is no more similar to $Y$ than $X^{\prime}$ is similar to $Y^{\prime}$. It is supposed to be provided by experts of the field, for instance economists. Beyond this qualitative assessment of similarities, we propose a quantitative evaluation of similarities through a similarity measure $S: \mathcal{Y}^{2} \rightarrow \mathbb{R}$. We consider that $S$ represents $\preccurlyeq$ if and only if for every $(X, Y),\left(X^{\prime}, Y^{\prime}\right) \in \mathcal{Y}^{2}$ :

$$
(X, Y) \preccurlyeq\left(X^{\prime}, Y^{\prime}\right) \Longleftrightarrow S(X, Y) \leq S\left(X^{\prime}, Y^{\prime}\right)
$$

The question is then to choose an appropriate similarity measure compatible with the given comparative similarity. To answer this question, we limit our research of an appropriate similarity measure to the general class introduced by Tversky (1977) in the framework of measurement theory. In this setting, the similarity $S(X, Y)$ between two objects $X$ and $Y$ depends on the evaluation of elements common to $X$ and $Y$, and elements pertaining to only one of them. When the values of attributes are fuzzy, it is necessary to take into account the fuzzy definition of intersection, union and differences previously introduced, and to use the indicated measure $m$ to evaluate them.

One way to choose a similarity measure representing a given comparative similarity is to look at the main properties which are implicitly assumed by the expert of the field. To have a list of possible properties, we can, for instance, think of basic ones, such as reflexivity, symmetry or transitivity, as similarities are softened versions of classic equivalences. Tversky claimed that transitivity is not necessarily requested from similarities.

We recall well-known similarity measures, such as the one originally introduced in Jaccard (1908) and defined as: $S^{1}(X, Y)=\frac{m(X \cap Y)}{m(X \cup Y)}$, or the one introduced in Ochiai (1957) as $S^{2}(X, Y)=\frac{m(X \cap Y)}{\sqrt{m(X)} \cdot \sqrt{m(Y)}}$.

They satisfy a property of symmetry. Neighbouring forms are frequently used as $S^{3}(X, Y)=\frac{m(X \cap Y)}{m(Y)}$ and $S^{4}(X, Y)=\frac{m(X \cap Y)}{m(X)}$. They are not based on a symmetry between $X$ and $Y$, but on the comparison of an object $Y$ with a reference $X$, which makes a clear difference between them, $S^{3}$ being regarded as a measure of satisfiability of $Y$ with regard to $X$ and $S^{4}$ as a measure of inclusion of $Y$ in $X$, as pointed out in Bouchon-Meunier et al. (1996).

\subsection{Axioms for comparative similarities}

More generally, we present axioms that may or may not be imposed on comparative similarities, stemming from measurement theory (Luce et al, 1990). Their formal definition is given in Coletti and Bouchon-Meunier (2019a) and developed in Coletti and Bouchon-Meunier (2020). 
Axiom FSO (weak order) is the most simple and it states that the comparative similarity $\preccurlyeq$ is a weak order on $\mathcal{Y}^{2}$.

Axiom FS1G (boundary min-condition) states that the similarity between two objects with no common attributes is always smaller than the similarity between objects which have some characteristics at least partly in common.

Axiom FS2G (weak boundary condition) assumes the following: in pairs of objects such that the degree of membership of the attributes in common is null, the degree of membership of the attributes present in only one object does not influence the degree of similarity. Analogously, in pairs with no attribute present in only one object, the degree of similarity is not influenced by the presence of more or less attributes in common.

Axiom FS3 (symmetry) declares that the similarity does not depend on the order of objects.

Axiom FS4 (partial attribute uniformity) says that all the attributes play the same role with respect to the similarity degree. This means that, if in both elements of a pair, the degrees of membership of two attributes are exchanged, a pair similar to the previous one is obtained.

Axiom FS4G (partial attribute stability) claims that the comparative degree of similarity of a pair of objects does not change if we slightly alter the values of membership of two attributes, the alteration being limited according to the difference between their values.

Axiom FS5 (distinctive attribute interchangeability) says that the degree of similarity is the same for a pair $(X, Y)$ and a pair $\left(X^{\prime}, Y^{\prime}\right)$ which differ by the presence and absence of an attribute present in $X$, but not in $Y$, or absent in $X^{\prime}$ and present in $Y^{\prime}$.

Axiom FS5G (asymmetric compensation) states that the degree of similarity between two objects remains the same when we slightly increase the membership of an attribute strongly present in one of them and we slightly decrease the membership of an attribute strongly present in the other one.

Axiom FS6G (asymmetric min-robustness) assumes that the degree of similarity between two objects remains the same when the membership of an attribute more strongly present in one of them than in the other one is slightly changed.

Axiom FS7 $7_{1}$ (monotonicity) considers that the greater the measure of the intersection and the smaller the measure of the difference between two objects, the higher their comparative similarity.

Axiom $\mathrm{FST}_{2}$ (weak monotonicity) considers that the greater the measure of the intersection and the smaller the measure of the attributes present in only one of the objects, the higher their comparative similarity.

\section{Classes of similarities}

Several of these available axioms can look natural to the expert of the domain. It is therefore interesting to see if we can identify classes of similarity measures satisfying them, to be able to represent the qualitative comparative similarity expressed by the expert by a quantitative similarity measure. We focus on well-known general classes of similarity measures.

\subsection{Representation of comparative similarities}

To the mentioned axioms, we add the Debreu condition $Q$ (Debreu (1954), regarding the representation of any order by a real function. The following equivalence between condition (1) and condition (2) can then be proved for any comparative similarity $\preccurlyeq$ on $\mathcal{Y}^{2}$.

(1) $\preccurlyeq$ satisfies FSO, FS1G, FS2G, FS4, FS4G, FS5, FS5G, FS6G, FS7 1 and Q.

(2) There exists a (unique under increasing transformations) function $S_{1}: \mathcal{Y}^{2} \rightarrow[0,1]$ representing $\preccurlyeq$ and such that:

- $S_{1}(X, Y)=\Phi[m(X \cap Y), m(X \Delta Y)]$;

- $\Phi(u, v)$ is increasing with respect to $u$ and decreasing with respect to $v$; and

- $\Phi(0, b)=0$, and $\Phi(a, 0)=1$, for every $a$ and for every $b \neq 0$. 
AJEB

4,3

It means in particular that, if the expert accepts the axioms listed in condition (1), then we can represent his/her comparative similarity by means of a similarity measure of the form $S_{1}$. Moreover, if he/she refuses one of these properties, then we cannot use such a similarity measure. It can be noted that $S^{1}$ is an example of similarity measure $S_{1}$.

If we consider another general class of similarity measures, we can also prove the equivalence between the following conditions (3) and (4) defined for any comparative similarity $\preccurlyeq$ on $\mathcal{Y}^{2}$.

(3) $\preccurlyeq$ satisfies FSO, FS1G, FS2G, FS4, FS4G, FS6G, FS7 2 and Q.

(4) There exists a (unique under increasing transformations) function $S_{2}: \mathcal{Y}^{2} \rightarrow[0,1]$ representing $\preccurlyeq$ and such that:

- $S_{2}(X, Y)=\Psi[m(X \cap Y), m(X \cap Y)+m(X, Y), m(X \cap Y)+m(Y, X)]$;

- $\Psi(u, v, w)$ is increasing with respect to $u$ and decreasing with respect to $v$ and $w$; and

- $\Psi\left(0, b, b^{\prime}\right)=0$ and $\Psi(a, 0,0)=1$, for every $a \neq 0$ and for every $b, b^{\prime}$.

In particular, if the expert accepts the axioms listed in condition (3), then his/her comparative similarity can be represented by a similarity measure of the form $S_{2}$. If he/she refuses one of them, then such a similarity measure cannot be used to represent a given comparative similarity. It can be noted that $S^{2}, S^{3}$ and $S^{4}$ are examples of similarity measure $S_{2}$.

\subsection{Specific classes of similarity measures}

The classes of similarity measures $S_{1}$ and $S_{2}$ being very general, we consider the more specific ones, defined as follows:

$$
S_{f, g}(X, Y)=\left\{\begin{array}{l}
\frac{f(m(X \cap Y))}{f(m(X \cap Y))+g(m(X \Delta Y))} \\
\text { for } m(X \cap Y)+m(X \Delta Y) \neq 0 \\
0
\end{array}\right. \text { otherwise }
$$

where $f$ and $g$ are nonnegative increasing real functions with $f(0)=g(0)=0$. When $f$ and $g$ are the identity function, we see that $S_{f_{g}}$ is a fuzzified version of $S^{1}$.

$$
T_{\delta, \gamma}(X, Y)= \begin{cases}\frac{\delta(m(X \cap Y)) \gamma(m(X \cap Y))}{\delta(m[(X \cap Y)+m(X \backslash Y)]) \gamma(m[(X \cap Y)+m(Y \backslash X)])} & \text { otherwise } \\ \text { for } m(X \cap Y), m(X \backslash Y)], m(Y \backslash X) \neq 0 & \\ 0 & \end{cases}
$$

where $\delta$ and $\gamma$ are nonnegative increasing real functions with $\delta(0)=\gamma(0)=0$. When $\delta$ and $\gamma$ are the square root function, we observe that $T_{\delta, \gamma}$ is a fuzzified version of $S^{2}$. When $\delta(x)=$ 1 for all $x$, and $\gamma$ is the identity function, then $T_{\delta, \gamma}$ is a fuzzified version of $S^{3}$. When $\gamma(x)=1$ for all $x$, and $\delta$ is the identity function, then $T_{\delta, \gamma}$ is a fuzzified version of $S^{4}$.

\section{Independence axioms}

To follow Tversky's approach of similarities requests to take into account a property of independence that we can revisit in a soft manner as follows, denoting by $\underline{0}$ the element of $\mathcal{Y}^{2}$ with all components equal to 0 : 
Axiom FWI (weak independence) states that, for every $\left(X_{1}, Y_{1}\right),\left(X_{2}, Y_{2}\right),\left(Z_{1}, W_{1}\right),\left(Z_{2}, W_{2}\right)$ $\in \mathcal{Y}^{2}$, if one of the following conditions holds:

- $X_{i} \cap Y_{i},=Z_{i} \cap W_{i}(i=1,2)$ and $X_{1} \Delta Y_{1}=X_{2} \Delta Y_{2} \neq \underline{0}, Z_{1} \Delta W_{1}=Z_{2} \Delta W_{2} \neq \underline{0}$

- $X_{i} \Delta Y_{i},=Z_{i} \Delta W_{i}(i=1,2)$ and $X_{1} \cap Y_{1}=X_{2} \cap Y_{2} \neq \underline{0}, Z_{1} \cap W_{1}=Z_{2} \cap W_{2} \neq \underline{0}$

then $\left(X_{1}, Y_{1}\right) \preccurlyeq\left(X_{2}, Y_{2}\right) \Longleftrightarrow\left(Z_{1}, W_{1}\right) \preccurlyeq\left(Z_{2}, W_{2}\right)$.

In the case where more such conditions are satisfied, a stronger version of independence is identified in the following axiom.

Axiom FCI (cumulative independence) establishes sufficient conditions (Coletti and Bouchon-Meunier, 2019a, 2020) for the equivalence: $\left(X_{1}, Y_{1}\right) \preccurlyeq\left(Z_{1}, W_{1}\right) \Longleftrightarrow\left(X_{2}, Y_{2}\right) \preccurlyeq\left(Z_{2}\right.$, $\left.W_{2}\right)$.

We can then characterise the class of similarity measures $S_{f g}$ under the umbrella of independence, proving that, if a comparative similarity $\preccurlyeq$ is represented by a similarity measure of the form $S_{f g}$, then it means that $\preccurlyeq$ satisfies $F S O, F S 1 G, F S 2 G, F S 3, F S 4, F S 4 G$, $F S 5, F S 5 G, F S 6 G, F S 7_{1}$ and $F W I$.

If one of these axioms is not acceptable to the expert expressing the comparative similarity, then it is impossible to represent it by a similarity measure of the form $S_{f_{g}}$.

In an analogous manner, we can characterise the class of similarity measures $T_{\delta, \gamma}$ by considering a property of independence. We can prove that, if a comparative similarity $\preccurlyeq$ is represented by a similarity measure of the form $T_{\delta, \gamma}$, then $\preccurlyeq$ satisfies $F S O, F S 1 G, F S 2 G$, $F S 4, F S 4 G, F S 6 G, F S 72$ and $F C I$.

Again, if the expert refuses any of these axioms, then the comparative similarity he expresses cannot be represented by a similarity measure of the form $T_{\delta, \gamma}$.

\section{Illustrative example}

As an example, we report here the sketch of a procedure for finding the most appropriate measure of similarity, expressing the idea of "no more similar than" of an expert, referring to a bank and the relevant study of the numerical measure agreeing with his/her comparative similarity. This procedure can be implemented by combining two different methodologies: presenting direct explicit queries on the simplest axioms and preparing simple fictitious pairs of bank customers applying for a loan, focusing on some axioms and requiring the expert to compare these pairs in "similarity". This last method permits to test which axioms are violated and which are accepted in the expressed similarity ordering.

To this end, the plan is to ask the bank staff of experts to prepare a database consisting in profiles of customers. The experts are required to assign degrees in $[0,1]$ to a set of characteristics to indicate that they are present to a certain extent. In this way, they create profiles closer to the reality and able to contribute in a more subtle way than a binary identification of the characteristics, to the elicitation of similarities between companies.

We refer to the example sketched in Section 2.2 and we submit to a bank staff a set of profiles related to possible companies applying for a loan, characterised by the attributes $h_{i}(i=1, \ldots, 10)$.

Consider now a data set consisting in the following profiles:

Each expert is asked to provide a binary relation among pairs of profiles, expressing his/her opinion about the fact that the elements of a pair are no more similar than the elements of another pair.

Firstly, his/her complete acceptance of the transitivity of this relation must be tested, explaining that no real function can represent a comparative similarity violating transitivity.

On the contrary, the structural axioms, having essentially a syntactical role because of the representability of any relation defined on an uncountable set, and so not related to the particular meaning of the binary relation, will not be submitted to the experts. 
AJEB 4,3
We now present just as an example, a sketch of the above process, using a scheme similar the one used in Coletti and Bouchon-Meunier (2019a, 2019b).

Questions about axioms $F S 1 G$ can reveal the possible propensity of the experts to use (min, max). More easily, it will be possible to discover if some of them have a clear attitude of

$44 \quad$\begin{tabular}{lllllllllll}
\hline$H$ & $h_{1}$ & $h_{2}$ & $h_{3}$ & $h_{4}$ & $h_{5}$ & $h_{6}$ & $h_{7}$ & $h_{8}$ & $h_{9}$ & $h_{10}$ \\
\hline$X_{1}$ & 0.1 & 0.3 & 0.2 & 0 & 0.1 & 0.01 & 0.2 & 0.5 & 0.2 & 0.1 \\
$X_{2}$ & 0.4 & 0.6 & 0.3 & 0.7 & 0.1 & 0.5 & 0.1 & 0.2 & 0.3 & 0.3 \\
$X_{3}$ & 0 & 0 & 0 & 1 & 0.8 & 0.9 & 1 & 0 & 0 & 0 \\
$X_{4}$ & 0.5 & 0.9 & 1 & 0 & 0 & 0 & 0 & 0.2 & 0.5 & 0.4 \\
$X_{5}$ & 0.2 & 0.1 & 0.1 & 0 & 0.2 & 0.3 & 0.01 & 0.2 & 0.1 & 0.5 \\
$X_{6}$ & 0.3 & 0.4 & 0.1 & 0.7 & 0.1 & 0.6 & 0.5 & 0.3 & 0.3 & 0.2 \\
$X_{7}$ & 0.5 & 0.4 & 0.1 & 0.6 & 0 & 0.7 & 0.8 & 0.3 & 0.5 & 0.2 \\
$X_{8}$ & 0.5 & 0.4 & 0.05 & 0.6 & 0.05 & 0.9 & 0.6 & 0.3 & 0.5 & 0.2 \\
$X_{9}$ & 0.4 & 0.8 & 0.3 & 0.8 & 0.1 & 0 & 0.1 & 0.4 & 0.1 & 0.6 \\
$X_{10}$ & 0.4 & 0.6 & 0.3 & 0.7 & 0.1 & 0.5 & 0 & 0.2 & 0.3 & 0.3 \\
$X_{11}$ & 0.4 & 0.1 & 0.01 & 0.2 & 0.1 & 0 & 0.2 & 0.5 & 0.2 & 0.1 \\
$X_{12}$ & 0.4 & 0.1 & 0.3 & 0.4 & 0.2 & 0.6 & 0.1 & 0.2 & 0.3 & 0.5 \\
$X_{13}$ & 0.1 & 0.6 & 0.2 & 0.7 & 0.9 & 0.01 & 0.2 & 0.7 & 0.1 & 0.8 \\
$X_{14}$ & 0.6 & 0.3 & 0.3 & 0 & 0.1 & 0.5 & 0.1 & 0.6 & 0 & 0.9 \\
$X_{15}$ & 0.4 & 0.6 & 0.01 & 0.2 & 0.1 & 0 & 0 & 0.7 & 0.1 & 0.8 \\
$X_{16}$ & 0.6 & 0.1 & 0.3 & 0.7 & 1 & 0.5 & 0.2 & 0.6 & 0 & 0.9 \\
\hline
\end{tabular}

rejection. To achieve this, we present to the expert the pairs $\left(X_{1}, X_{2}\right)$ and $\left(X_{3}, X_{4}\right)$, to which the relation $\left(X_{3}, X_{4}\right) \prec\left(X_{1}, X_{2}\right)$ must be assigned, if one accepts axiom $F S 1 G$.

In particular, the experts must consciously consider two profiles having no feature in common strictly less similar than any others, independently of the degree of membership of the common feature in these last ones. If he/she consciously assesses $\left(X_{3}, X_{4}\right) \sim\left(X_{1}, X_{2}\right)$, it will be necessary to consider an operator different from the Gödel's one (Coletti and Bouchon-Meunier, 2019a, 2019b).

Now we need to test the propensity of the expert to accept the axioms necessary for the representability of a comparative similarity by any element of the two classes considered in this paper (i.e. $S_{f_{g}}$ and $T_{\gamma, \delta}$ ), that is axioms $F S 4$ and $F S 4 G$. To achieve this, we first ask the expert to order by similarity the pairs $\left(X_{1}, X_{2}\right)$ and $\left(X_{5}, X_{6}\right)$, which only differ with respect to a permutation of the indices, and then the pairs $\left(X_{7}, X_{9}\right)$ and $\left(X_{8}, X_{9}\right)$, which are such that $X_{8}$ is obtained from $X_{7}$ by subtracting 0.005 to the degree of belonging of the characteristic $h_{3}$ and adding it to degree of belonging of the characteristic $h_{5}$ and subtracting 0.2 to the degree of belonging of the characteristic $h_{7}$ and adding it to degree of belonging of the characteristic $h_{6}$.

If he/she does not consciously consider either the first two pairs equally similar (violating in this way axiom FS4), or the second two equally similar (violating in this way axiom FS4G), then there is no similarity measure considered in this paper representing his/her comparative similarity.

On the contrary, in the case where the judgement is $\left(X_{1}, X_{2}\right) \sim\left(X_{5}, X_{6}\right)$ and $\left(X_{7}, X_{9}\right) \sim\left(X_{8}, X_{9}\right)$, we only need to explain that the equivalence must not be casual, but must be based on the awareness of the equal contribution of the attributes to the similarity and the comparative degree of similarity of a pair does not change if we "slightly" alter the values of membership of two attributes.

If the experts agree, we can proceed with the process of discovering the most suitable similarity measure representing their idea of "no more similar than". 
At this point, the following pairs $\left[\left(X_{1}, X_{2}\right),\left(X_{10}, X_{11}\right)\right]$ are submitted for a comparative evaluation in similarity, by putting in evidence that the two pairs are such that the total degree of the common "presence" of characteristics (i.e. $m\left(X_{r} \cap Y_{r}\right)=\sum_{i} \min \left(x_{i}, y_{i}\right)$ ) is Fuzzy
similarity
measure equal, such as the total degree of the distinctive "presence" of characteristics, computed as the maximum between the two values obtained as minimum between the degree of "presence" in one company and the degree of "not presence" in the others (i.e. $\left.m\left(X_{i} \Delta Y_{i}\right)=\sum_{i} \max \left\{\min \left(x_{i}, 1-y_{i}\right), \min \left(y_{i}, 1-x_{i}\right)\right\}\right)$.

The fact that the experts do not consider the two pairs equally similar means that they do not accept at least one among the axioms FS5, FS6 and FS5G, and so no function $\Phi$ can represent their comparative similarity. At this point, it is necessary to discover which axioms are actually violated, as FS5 and FS6 are necessary for the representability by all the measures considered in the paper.

To this end, we propose to consider the three pairs $\left[\left(X_{1}, X_{2}\right),\left(X_{10}, X_{11}\right),\left(X_{11} \cap X_{12}\right)\right]$ which are such that $m\left(X_{11} \cap Y_{12}\right)>m\left(X_{10} \cap Y_{11}\right)=m\left(X_{1} \cap Y_{2}\right)$ and $m\left(X_{11} \Delta Y_{12}\right)=m\left(X_{1} \Delta Y_{2}\right)<m$ $\left(X_{10} \Delta Y_{11}\right)$ and require to order them in similarity.

If the expert provides the following ordering $\left(X_{10}, X_{11}\right) \prec\left(X_{1}, X_{2}\right) \prec\left(X_{11}, X_{12}\right)$, his/her comparative similarity does not violate axioms FSO, FS4, FS4G, FS5, FS5G and $F S 7_{1}$.

In this first step, we can discuss with the expert about the following fact: accepting axioms $F S O, F S 4, F S 4 G, F S 5, F S 5 G$ and $F S 7_{1}$ is, in fact, equivalent to accepting that the similarity between two companies is evaluated through a function only depending on the fuzzy cardinality of the degree of the common "presence" of characteristics $h_{i}$ [i.e. $m(X \cap Y)$ ] and the fuzzy cardinality of the degree of the global distinctive presence of the same characteristics [i.e. $m(X \Delta Y)$ ]; vice versa, to choose as a similarity measure, any function of such a large class means to accept all of the axioms mentioned above. Particular emphasis should be put on discussing axiom $F S 7_{1}$; clarifying that in the monotonicity, the role of the intersection is greater than that of the difference.

On the contrary, if the expert proposes a different order for the above pairs, then it is necessary to stress that no similarity measure of the kind discussed before is agreeing with his/her comparative similarity.

If the case of a positive agreement of the expert with the axioms, it remains to test which axioms determining a specific form of the function $\Phi$ are accepted and which are not. In this regard, it would be good to underline that the previous axioms fix some relations that essentially divide the possible profiles of companies into equivalence classes, but leave some degrees of freedom that are partially saturated by the axioms that identify the form of the function $\Phi$.

To this end, we must first ask him/her if his/her order between $\left(X_{13}, X_{14}\right)$ and $\left(X_{15}, X_{16}\right)$ is the same as the ordering between $\left(X_{1}, X_{2}\right)$ and $\left(X_{10}, X_{11}\right)$, about which he/she has already expressed the following order in similarity $\left(X_{10}, X_{11}\right) \prec\left(X_{1}, X_{2}\right)$. A negative answer violates axiom FWI and precludes the possibility to represent the expert's comparative similarity by any element of the class $S_{f, g}$.

If, on the contrary, the expert agrees with the proposed inequality, it is necessary to explain to him/her the actual meaning of the axiom of weak independence, by stressing the following: to refuse it underlines the idea that the characteristics $h_{i}$ cannot be considered independent, but their mutual influences (more precisely their positive or negative interactions) must be taken into account in the similarity evaluation.

A similar procedure can be implemented to test the agreement with the axioms related to the class of function $S_{2}$ and then to the class $T_{\delta, \gamma}$. We note that, at the basis of this class, there is the 
AJEB

4,3

fact that all the characteristics $h_{i}$ have the same importance. If this is considered not realistic, it is necessary to change the class of measures, and so the set of axioms.

\section{Comparative dissimilarities}

When similarities are used to evaluate to which extent an object is typical of a category to define prototypes, they are generally associated with dissimilarities, according to Rosch (1978), Rosch and Mervis (1975) and Prasetyo and Purwarianti (2014).

In this setting, an object is typical of its category if it is highly similar to other objects of this category and highly dissimilar to objects of other categories, a prototype corresponding to the highest degrees. General classes of dissimilarities have been pointed out in Bouchon-Meunier et al. (1996) and analysed in various papers, for instance Goshtasby (2012). It is very common to use distances to represent dissimilarities, but they are not the only possibilities. Dissimilarities should not be considered as the opposite of similarities, as they do not necessarily satisfy the same properties as similarities or their opposites. When dissimilarities are assigned by an expert in a way similar to the previous approach of similarities, we need to look at a convenient dissimilarity measure to represent it numerically (Coletti et al., 2020).

We explore the representation of a so-called comparative dissimilarity between objects described by means of imprecise or linguistic values of attributes, defined as a binary relation on $\mathcal{Y}^{2}$ denoted by $\preccurlyeq^{\prime}$ such that, for two pairs of objects $(X, Y)$ and $\left(X^{\prime}, Y^{\prime}\right) \in \mathcal{Y}^{2},(X, Y)$ $\preccurlyeq^{\prime}\left(X^{\prime}, Y^{\prime}\right)$ means that $X$ is no more dissimilar to $Y$ than $X^{\prime}$ is dissimilar to $Y^{\prime}$. Moreover, $(X, Y) \prec^{\prime}\left(X^{\prime}, Y^{\prime}\right)$ stands for $(X, Y) \preccurlyeq{ }^{\prime}\left(X^{\prime}, Y^{\prime}\right)$ and $\operatorname{not}\left(X^{\prime}, Y^{\prime}\right) \preccurlyeq{ }^{\prime}(X, Y)$.

We then consider that a dissimilarity measure $D: \mathcal{Y}^{2} \rightarrow R$ represents the comparative dissimilarity $\preccurlyeq^{\prime}$ if and only if, for all $(X, Y),\left(X^{\prime}, Y^{\prime}\right) \in Y^{2}$, it holds that

$$
\begin{aligned}
& (X, Y) \preccurlyeq^{\prime}\left(X^{\prime}, Y^{\prime}\right) \Rightarrow D(X, Y) \leq D\left(X^{\prime}, Y^{\prime}\right), \\
& (X, Y) \prec^{\prime}\left(X^{\prime}, Y^{\prime}\right) \Rightarrow D(X, Y)<D\left(X^{\prime}, Y^{\prime}\right) .
\end{aligned}
$$

With the purpose of characterising dissimilarity measures able to represent a given qualitative comparative dissimilarity, we propose a list of axioms that may or may not be satisfied by a dissimilarity measure, and that the expert providing the comparative dissimilarity can consider natural or not.

Axiom FDO (weak order) states that $\preccurlyeq^{\prime}$ is a weak order on $\mathcal{Y}^{2}$.

Axiom FD1 (incremental independence) requires the comparative degree of dissimilarity to be independent of the common (positive or negative) increment of presence/absence of the features in the objects of a pair.

Axiom FD2 (local strong symmetry) requires that, when we exchange the values of one of the attributes in two objects, the dissimilarity between the objects is not changed.

Axiom FD3 (boundary condition) claims that an object is never less dissimilar to another object than it is to itself. An object is never more dissimilar to another object than it is to its complement if and only if it is crisp, with values of attributes in $[0,1]$.

Axiom FD4 (monotonicity axiom) claims that we increase the dissimilarity between $X$ and $Y$ by reducing for $X$ the value of an attribute which is less present in $X$ than in $Y$, or by increasing in $Y$ the value of the same attribute.

Considering again the Debreu condition in addition to the considered axioms, and the classic inclusion of fuzzy sets $\subseteq$, the following conditions (5) and (6) can then be proven equivalent for a comparative similarity $\preccurlyeq^{\prime}$ on $\mathcal{Y}^{2}$.

(6) $\preccurlyeq$ ' satisfies axioms FD1, FD2, FD3, FD4 and $D$. 
(7) There exists a function (unique under increasing transformations) $\Phi: \mathcal{Y}^{2} \rightarrow[0,1]$ representing $\preccurlyeq$ ' and a function $\varphi: \mathcal{Y} \rightarrow \mathbb{R}$ such that:

- $Z \subseteq Z^{\prime} \Rightarrow \varphi(Z) \leq \varphi\left(Z^{\prime}\right)$, for every $Z, Z^{\prime} \in \mathcal{Y}$;

- $\varphi(\underline{0})=0$ and $\varphi(\underline{1})=1$; and

- for all $X, Y \in Y: \Phi(X, Y)=\Phi(|X-Y|, \underline{0})=\varphi(|X-Y|)$.

A classic example of dissimilarity measure is the weighted Manhattan distance, defined for any set of parameters $\alpha=\left(\alpha_{1}, \ldots, \alpha_{p}\right)$ with $\alpha_{k} \geq 0$ and $\sum_{k=1}^{p} \alpha_{k}=1$, as a function $D_{a}: \mathcal{Y}^{2} \rightarrow$ $\mathbb{R}$ defined, for every $X, Y \in \mathcal{Y}$, as:

$$
D_{\alpha}(X, Y)=\sum_{k=1}^{p} \alpha_{k}\left|x_{k}-y_{k}\right| .
$$

It can be proven that such a distance satisfies axioms FD1, FD2, FD3 and FD4. Therefore, if the expert does not accept any of these axioms, it is not possible to use a weighted Manhattan distance to represent his/her comparative dissimilarity. More generally, other kinds of distances can be analysed in this framework (Coletti et al., 2020). An experiment similar to the sketch of the illustrative example presented for similarities could enable us to know how to represent the qualitative comparative dissimilarity expressed by the expert.

\section{Conclusion}

The purpose of this paper is to present in a simple formulation the methodology to represent by numerical measures the qualitative similarities, and to a certain extent, the qualitative dissimilarities, expressed by experts. Such similarity or dissimilarity measures are usually chosen arbitrarily among the long list of possibilities, without checking if they correspond to the expert's interpretation of his/her spontaneous evaluations. It is therefore important to interact with him/her to find out what kind of properties he/she considers to be natural in terms of the behaviour of the similarity or dissimilarity he/she expresses.

There is a large number of similarity measures in the classic case and they give rise to an even larger set of quantities in the case where the attribute values describing the objects to compare are fuzzy. We have focused on the specific Gödel operators on fuzzy sets because they are the most commonly used, but more results can be found in the authors' recent papers. Several classes of similarity and dissimilarity measures have been considered and similar work can be done on other general classes.

\section{References}

Basel Committee on Banking Supervision (2001), "Overview of the new Basel capital accord", available at: www.bis.org/publ/bcbsca02.pdf/

Basel Committee on Banking Supervision (2017), "Basel ii: international convergence of capital measurement and capital standards: a revised framework", available at: www.bis.org/publ/bcbs107.htm

Bouchon-Meunier, B., Coletti, G., Lesot, M.J. and Rifqi, M. (2009), "Towards a conscious choice of a similarity measure: a qualitative point of view", in Claudio S. and Gaetano, C. (Eds), Symbolic and Quantitative Approaches to Reasoning with Uncertainty, Springer Berlin Heidelberg, Berlin, pp. 542-553.

Bouchon-Meunier, B., Coletti, G., Lesot, M.-J. and Rifqi, M. (2010), "Towards a conscious choice of a fuzzy similarity measure: a qualitative point of view", in Hüllermeier, E., Kruse, R. and Hoffmann, F., (Eds), Computational Intelligence for Knowledge-Based Systems Design, Springer Berlin Heidelberg, Berlin, pp. 1-10. 
Bouchon-Meunier, B., Rifqi, M. and Bothorel, S. (1996), “Towards general measures of comparison of objects", Fuzzy Sets and Systems, Vol. 84 No. 2, pp. 143-153.

Chen, S.M., Yeh, M.S. and Hsiao, P.Y. (1995), "A comparison of similarity measures of fuzzy values", Fuzzy Sets and Systems, Vol. 72 No. 1, pp. 79-89.

Coletti, G. and Bouchon-Meunier, B. (2019a), "Fuzzy similarity measures and measurement theory", In IEEE International Conference on Fuzzy Systems (FUZZ-IEEE), New Orleans, pp. 1-7.

Coletti, G. and Bouchon-Meunier, B. (2019b), "A study of similarity measures through the paradigm of measurement theory: the classic case", Soft Computing, Vol. 23 No. 16, pp. 6827-6845.

Coletti, G. and Bouchon-Meunier, B. (2020), "A study of similarity measures through the paradigm of measurement theory: the fuzzy case", Soft Computing, Vol. 24 No. 15, pp. 11223-11250.

Coletti, G., Petturiti, D. and Bouchon-Meunier, B. (2020), "A measurement theory characterization of a class of dissimilarity measures for fuzzy description profiles", in Lesot, M.J, Vieira, S.M., Reformat, M.Z., Carvalho, J.P., Wilbik, A., Bouchon-Meunier, B. and Yager, R.R. (Eds), Information Processing and Management of Uncertainty in Knowledge-Based Systems - 18th International Conference, IPMU 2020, Lisbon, Portugal, June 15-19, 2020, Proceedings, Part II, volume 1238 of Communications in Computer and Information Science, Springer, pp. 258-268.

Costas, P. and Nikos, I.K. (1993), “A comparative assessment of measures of similarity of fuzzy values”, Fuzzy Sets and Systems, Vol. 56 No. 2, pp. 171-174.

Debreu, P. (1954), Representation of Preference Ordering by a Numerical Function, Wiley, New York, NY.

Fan, J. and Xie, W. (1999), "Some notes on similarity measure and proximity measure", Fuzzy Sets and Systems, Vol. 101 No. 3, pp. 403-412.

Goshtasby, A.A. (2012), Similarity and Dissimilarity Measures, Springer London, pp. 7-66.

Jaccard, P. (1908), "Nouvelles recherches sur la distribution florale”, Bulletin de la Societe Vaudoise Des Sciences Naturelles, Vol. 44, pp. 223-270.

Lee-Kwang, H., Song, Y.-S. and Lee, K.-M. (1994), "Similarity measure between fuzzy sets and between elements”, Fuzzy Sets and Systems, Vol. 62 No. 3, pp. 291-293.

Luce, R.D., Suppes, P., Krantz, D.H. and Tversky, A. (1990), Foundations of Measurement, Academic Press, San Diego.

Ochiai, A. (1957), "Zoogeographic studies on the soleoid fishes found in Japan and its neighbouring regions", Nippon Suisan Gakkaishi, Vol. 22 No. 9, pp. 526-530.

Prasetyo, H. and Purwarianti, A. (2014), "Comparison of distance and dissimilarity measures for clustering data with mix attribute types", In 2014 The 1st International Conference on Information Technology, Computer, and Electrical Engineering, pp. 276-280.

Rosch, E. (1978), Principles of Categorization, Lawrence Erlbaum associates, pp. 27-48.

Rosch, E. and Mervis, C. (1975), "Family resemblance: studies of the internal structure of categories", Cognitive Psychology, Vol. 7 No. 4, pp. 573-605.

Tversky, A. (1977), "Features of similarity”, Psychological Review, Vol. 84 No. 4, pp. 327-352.

Wang, X., Baets, B.D. and Kerre, E. (1995), “A comparative study of similarity measures”, Fuzzy Sets and Systems, Vol. 73 No. 2, pp. 259-268.

Zadeh, L.A. (1971), "Similarity relations and fuzzy orderings", Information Sciences, Vol. 3 No. 2, pp. $177-200$.

Corresponding author

Bernadette Bouchon-Meunier can be contacted at: bernadette.bouchon-meunier@lip6.fr

For instructions on how to order reprints of this article, please visit our website:

www.emeraldgrouppublishing.com/licensing/reprints.htm

Or contact us for further details: permissions@emeraldinsight.com 